\title{
Standoff Raman Spectroscopy of C4 Explosive and Study the Effect of Integration Time and Laser Power
}

\author{
Mohammad R. Mohammad ${ }^{1}$, Haider G. Abdulzahraa ${ }^{1 *}$ and Naseer M. Hadi ${ }^{2}$ \\ ${ }^{1}$ Applied Science Department, University of Technology-Baghdad, Laser Research Centre, \\ ${ }^{2}$ Ministry of Science and Technology, Baghdad. \\ *Corresponding Author: spectrophysics84@ gmail.com
}

\begin{abstract}
In this paper standoff Raman spectroscopy SRS system for explosive materials is developed. Standoff Raman detection of C4 substance under dark laboratory condition at $4 \mathrm{~m}$ distance is achieved. A frequency doubled Nd:YAG laser at $532 \mathrm{~nm}$ excitation is used. The Raman scattered light is collected by a telescope and then transferred via fiber optics cable to spectrograph and finally into $\mathrm{CCD}$ detector. Notch filter used to reject Rayleigh scattering light. Carbon tetrachloride $\mathrm{CCL}_{4}$ and Acetone $\left(\mathrm{CH}_{3}\right)_{2} \mathrm{CO}$ are used as a calibration standard for the Raman measurements because of their strong and intensive scattering capability. Raman measurement of $\mathrm{C} 4$ explosive is also acquired using conventional Raman microscopy for verification of standoff Raman measurements. The effects of integration time and laser power on Raman cross section under dark condition were studied. Standoff Raman detection of C4 substance at $4 \mathrm{~m}$ distance under partially illuminated condition has been achieved and hence the effect of higher integration time was studied under the same condition. [DOI: 10.22401/JNUS.20.2.11]
\end{abstract}

Keywords: standoff Raman spectroscopy, explosive materials, notch filter, CCD detector.

\section{Introduction}

Raman spectroscopy is a powerful noncontact technique that uses a laser to probe the vibrational energy levels of molecules in a substance. The vibration information provided by a Raman spectrum is very specific for the chemical composition of the molecules [1]. Due to its chemical specificity, capability in analyzing and identifying organic and inorganic compounds in any state, nondestructive and, with little if any sample preparation required, Raman spectroscopy is becoming a promising remote detection tool for explosives identification and analysis purposes. Standoff Raman spectroscopy SRS which is an inelastic scattering of light with the same principles as conventional Raman spectroscopy is a technique that allows the detector to be away from the sample that is being studied. The demand for rapid field detection has led to an increase in development of commercial-portable Raman systems with more sensitive detectors and spectrometers operating with high power lasers.

During recent years, the world has been suffering many terrorist attacks by use of explosives and chemical warfare agents. In order to screen passengers and luggage fast and reliably, it is of significant importance to have a good technology for detecting high explosives HEs and analyzing the chemical identification accurately [2].

The aim of this research is to design a continuous wave standoff Raman spectroscopy system and to demonstrate its capability at laboratory scale as a tool in standoff detection technology. The standoff Raman system was tested in detection of $\mathrm{C} 4$ explosive while $\mathrm{CCL}_{4}$ and Acetone tested as calibration standards at distance of $4 \mathrm{~m}$ under dark conditions. One of the aims of the study is to study the influence of integration time and laser power on Raman signal. Standoff Raman detection of C4 substances was achieved also under partially illuminated environment in laboratory.

\section{Experimental Work}

The standoff Raman spectroscopy SRS system consists of a Cassegrain telescope with $110 \mathrm{~mm}$ clear aperture was used to collect relatively large area of Raman signals scattered from target sample, Cobolt Tor $^{\mathrm{TM}}$ laser system operating at $532 \mathrm{~nm}$ (frequency doubled Nd:YAG continuous wave laser) used as a excitation source for measuring Raman spectra of C4 explosive, Ventana-532-Raman spectrometer with $0.6 \mathrm{~nm}$ spectral resolution 
and grating $1600 \mathrm{~L} / \mathrm{mm}$ HD VPG, equipped with a Hamamatsu S11510 charge-coupled device (CCD) detector (Pixel format $1024 \mathrm{x}$ 64, Pixel size $14 \mu \mathrm{m}$ square), a THORLABS notch filter was adjusted to attenuate the elastically Rayleigh scattered light and diffuse reflected light from the target, a fiber optic assembly of $(600 \mu \mathrm{m}$ diameter, $\mathrm{NA}=0.39$ fiber input) and laptop computer.

The telescope was coupled to the Raman spectrometer through fiber optics cable. The notch filter was inserted between the telescope and the fiber optics. The output of the fiber optic bundle was directly coupled to the Raman spectrometer by $5 x$ objective lens. Once the laser strikes the sample, the reflecting telescope collects the scattered light from the sample that is being excited by the laser. The collimated light from the telescope output passing first through the notch filter and then the light is collected by fiber optic bundle. Finally, the light was directed into the CCD detector which is integrated in Ventana Raman spectrometer.

In order to verify the standoff Raman spectrum of $\mathrm{C} 4$ obtained by Ventana Raman spectroscopy system, Raman spectrum of the same substance has been recorded using a bench top Bruker SENTERRA Raman instrument which is considered as reference spectrum. Raman spectrum was recorded at $532 \mathrm{~nm}$ laser excitation with spectral resolution of $3 \mathrm{~cm}^{-1}$ in spectroscopic Raman shift range of $0-4270 \mathrm{~cm}^{-1}$. For measurements of explosives on surfaces, a known amount of the $\mathrm{C} 4$ explosive of $\mathrm{C} 4$ was wet with acetone and then the $\mathrm{C} 4$ substance was ground and pressed by spoon to be spread out and paste onto the glass background surface.

\section{Results and Discussion}

\section{Standoff Raman Spectra of Standard Samples}

Carbon tetrachloride $\mathrm{CCL}_{4}$ and Acetone $\left(\mathrm{CH}_{3}\right)_{2} \mathrm{CO}$ are typically used as a calibration standard for the Raman measurements, therefore they are used to test and evaluate the performance of the SRS system used in this study. They were chosen as the first samples to test because of their strong and intensive scattering capability. These compounds were transferred into quartz cuvette of $3.5 \mathrm{~mL}, 1 \mathrm{~cm}$ inside width. As shown in Fig.(1) standoff Raman spectra of standard samples were collected at a distance of $4 \mathrm{~m}$, in spectroscopic Raman shift range $(0-4270) \mathrm{cm}^{-1}$ using frequency doubled Nd:YAG $532 \mathrm{~nm} \mathrm{CW}$ laser with laser power of $250 \mathrm{~mW}$ and integration time (IT) of 2 second under dark laboratory conditions.

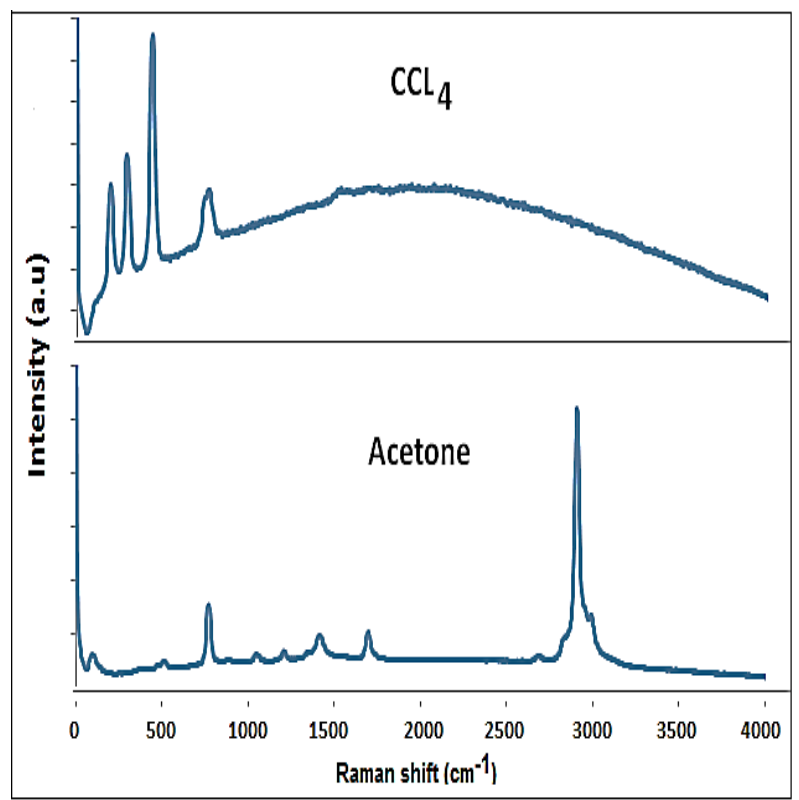

Fig.(1): Standoff Raman spectra of $\mathrm{CCL}_{4}$ and Acetone.

In Fig.(1) $\mathrm{CCL}_{4}$ shows its three main Raman active peaks at $(216,312,459) \mathrm{cm}^{-1}$. The most important characteristic band of $\mathrm{CCL}_{4}$ was found at $459 \mathrm{~cm}^{-1}$. In the case of Acetone, the fingerprint Raman bands are observed at $(390,507,786,1067,1226,1426$, $1709,2920,3001) \mathrm{cm}^{-1}$. The most prominent band of Acetone appeared at $786 \mathrm{~cm}^{-1}$ and $2918 \mathrm{~cm}^{-1}$ as clearly shown in the figure. All these fingerprint Raman bands of standard compounds are in very good agreement with previously published literature [3 - 5].

\section{Standoff Raman spectrum of Plastic Explosive C4}

Cyclotrimethylene-trinitramine, $\mathrm{C}_{3} \mathrm{H}_{6} \mathrm{~N}_{6} \mathrm{O}_{6}$. $\mathrm{C} 4$ contains a nitro group $\left(\mathrm{NO}_{2}\right)$. Fig.(2) shows standoff Raman spectrum of $\mathrm{C} 4$ collected at 4 $\mathrm{m}$ distance. This sample was detected using frequency doubled Nd:YAG $532 \mathrm{~nm}$ with laser power of $250 \mathrm{~mW}$ and the integration time of 2 seconds. The measurement has been performed on bulk amount sample, in the amount of $2 \mathrm{mg}$. The Raman signals were 
measured in the dark laboratory condition. Raman spectrum is contributed by background noise due to fluorescence and inherent optical noise of the detection Raman system.

Raman spectrum of $\mathrm{C} 4$ also measured by conventional Raman microscopy (which is known as micro Raman microscopy) that is used as a reference data for $\mathrm{C} 4$ measurement. Simple background reduction (baseline reduction) technique was applied to suppress these backgrounds of spectrum that is obtained by micro Raman microscopy while spectrum obtained by standoff Raman spectroscopy kept without any modification, it means there is no background reduction. The spectrum of $\mathrm{C} 4$ measured with micro Raman microscopy was depicted in Fig.(3) along with spectrum measured at standoff Raman spectroscopy. The most prominent Raman bands of $\mathrm{C} 4$ are assigned and listed in the Table (1) and the mode of vibrations are also indicated for each Raman band.

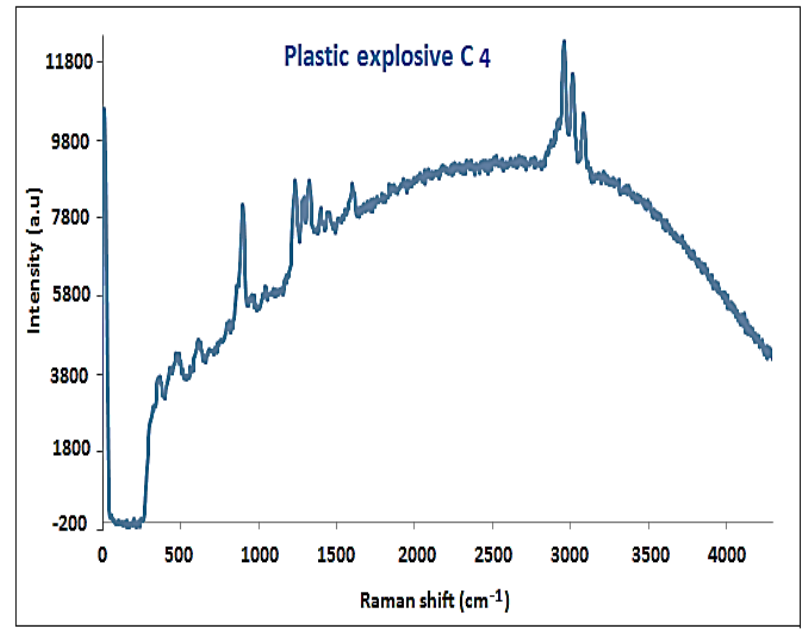

Fig.(2): C4 spectrum at $4 \mathrm{~m}, 250 \mathrm{~mW}$ and integration time of $2 \mathrm{~s}$.
Table (1)

The assignments of Raman band spectrum of C4.

\begin{tabular}{|c||c||}
\hline $\begin{array}{c}\text { Raman } \\
\left.\text { shift } \mathbf{( c m}^{-\mathbf{1}}\right)\end{array}$ & $\begin{array}{c}\text { Assignments } \\
\text { (mode of vibrations) }\end{array}$ \\
\hline \hline 885 & $\begin{array}{c}\text { C-N Symmetric ring- } \\
\text { breathing }\end{array}$ \\
\hline \hline 1217 & $\begin{array}{c}\text { N-N stretching and } \mathrm{NO}_{2} \\
\text { symmetric stretching }\end{array}$ \\
\hline 1274 & $\begin{array}{c}\text { N-N stretching and } \mathrm{NO}_{2} \\
\text { symmetric stretching }\end{array}$ \\
\hline \hline 1310 & $\begin{array}{c}\text { N-N stretching and } \mathrm{NO}_{2} \\
\text { symmetric stretching }\end{array}$ \\
\hline \hline 1390 & $\mathrm{CH}_{2}$ bending \\
\hline 1446 & $\mathrm{CH}_{2}$ bending \\
\hline 1590 & ONO stretching \\
\hline 2948 & C-H stretching \\
\hline \hline 3000 & C-H stretching \\
\hline 3072 & C-H stretching \\
\hline
\end{tabular}

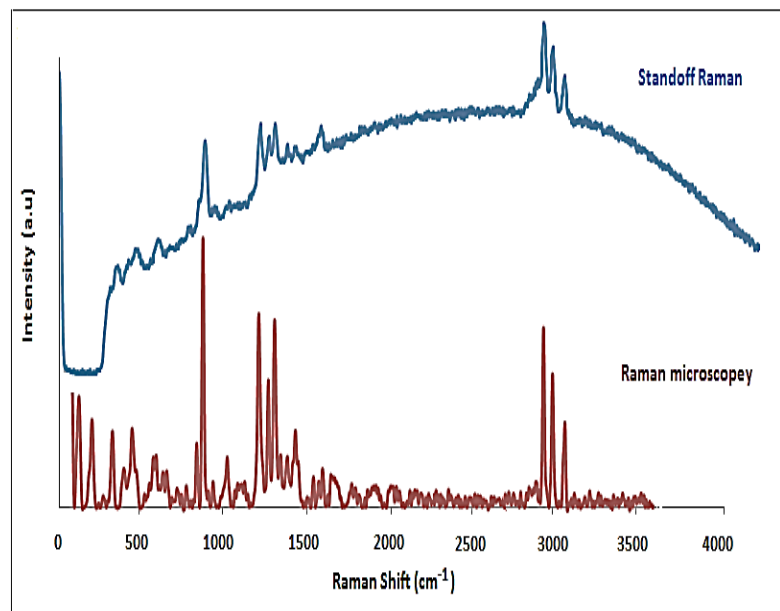

Fig.(3): Comparison of standoff Raman and micro Raman spectrum of C4.

The spectrum of $\mathrm{C} 4$ measured with standoff Raman spectroscopy is in a good agreement with spectra measured with micro Raman microscopy as obvious in Fig.(3) and the average difference in assigned peaks in spectral range $0-4270 \mathrm{~cm}^{-1}$ is about $2 \mathrm{~cm}^{-1}$. Standoff spectrum of $\mathrm{C} 4$ is in a good agreement with the spectrum obtained by other authors [5-9] and the average difference in assigned peaks in spectral range $0-4270 \mathrm{~cm}^{-1}$ is about $2 \mathrm{~cm}^{-1}$.

One of the aims of the study is to study the influence of integration time and laser power on Raman cross section and to determine the integration time and laser power at which the 
optimal signal to noise $(\mathrm{S} / \mathrm{N})$ ratio is achieved for standoff Raman spectra. As the number of inelastic scattered photons from the sample is increased, the Raman signal will be increased on the expanse of the noise, therefore it is said the $\mathrm{S} / \mathrm{N}$ ratio gets enhanced. The increasing in the number of scattered photons depends on the longer integration time and higher laser power.

\section{Integration Time Influence}

The time that the CCD detector takes to collect photons is known as the integration time or sometimes CCD integration time. Therefore, as the integration time increasing the number of inelastic scattered photons collected by CCD detector is increased as a result $\mathrm{S} / \mathrm{N}$ ratio significantly enhanced. In order to demonstrate this influence, to improve $\mathrm{S} / \mathrm{R}$ ratio and trigger the weak bands, the standoff Raman spectra of C4 substance were acquired for various integration times i.e. $400 \mathrm{~ms}, 1000 \mathrm{~ms}$ and $4000 \mathrm{~ms}$ and the remaining parameter conditions kept fixed with laser power of $250 \mathrm{~mW}$, standoff distance of $4 \mathrm{~m}$ and mass of $800 \mu \mathrm{g}$, and the measurements were acquired in dark condition. Firstly, Standoff measurements were taken for integration time of $400 \mathrm{~ms}$, as can be seen in Fig.(4) the Raman bands at 885, 2948, 3000 and $3072 \mathrm{~cm}^{-1}$ are observed while the remaining weak Raman bands in the spectroscopic region (1200- 1600) $\mathrm{cm}^{-1}$ are overmusked by background noise and becamed almost unnoticeable.

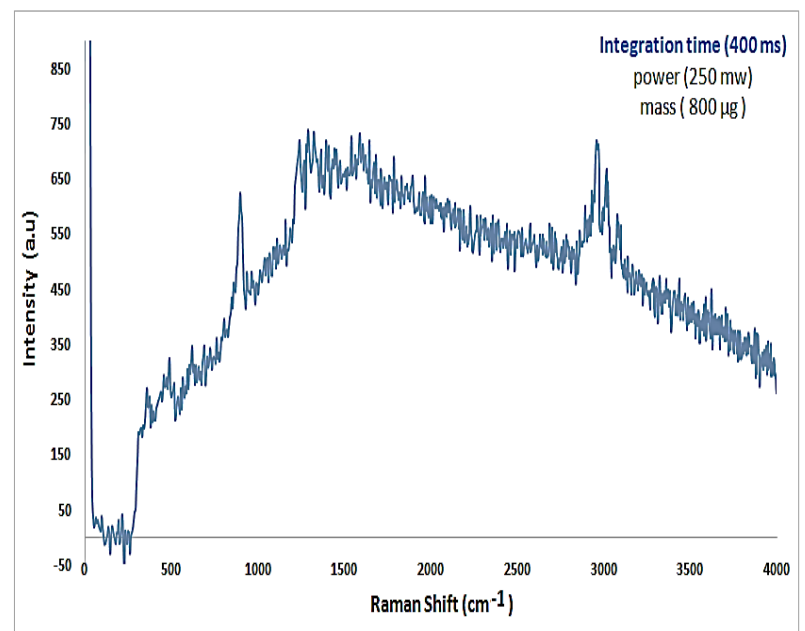

Fig.(4): Standoff Raman spectrum of C4 at integration time of $400 \mathrm{~ms}$.
The measurements were repeated for same condition but now with integration time of $1 \mathrm{~s}$, as clearly shown in Fig.(5), in addition to the enhancement in the prominent peaks, the higher integration time triggers additional weak Raman bands at 1217, 1274 and $1310 \mathrm{~cm}^{-1}$ but still such integration time was not sufficient to show the characteristic Raman bands at 1390, 1446 and $1590 \mathrm{~cm}^{-1}$ which are buried by the presence of the fluorescence.

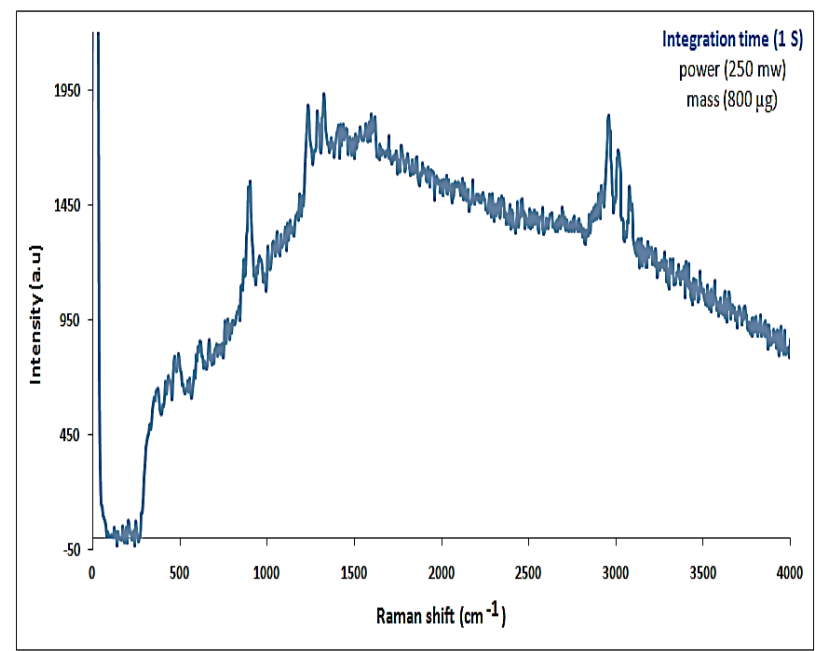

Fig.(5): Standoff Raman spectrum of C4 at integration time of $1 \mathrm{~s}$.

Finally, the integration time increased to be 4 second, as can be seen in Fig.(6) such integration time was sufficient to show the all ten Raman bands including the prominent bands and the weak bands.

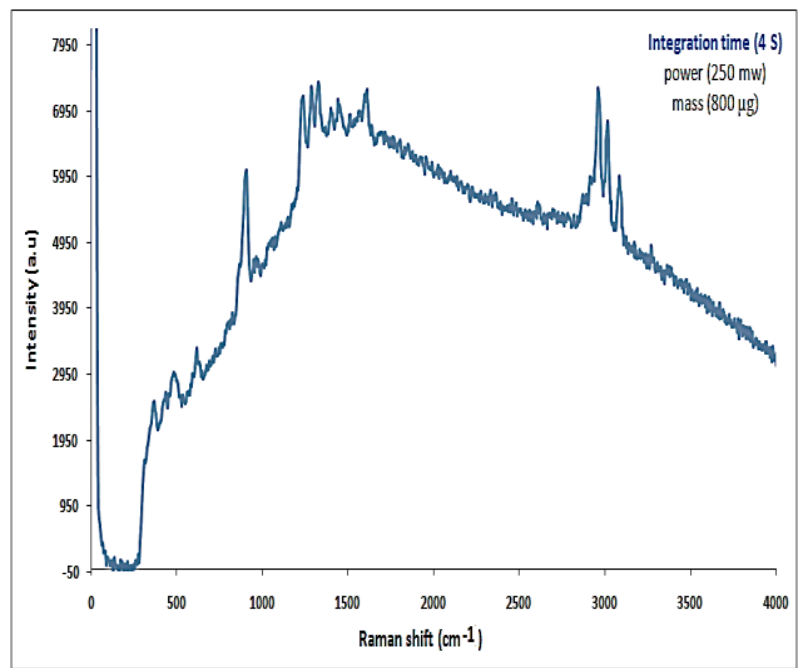

Fig.(6): Standoff Raman spectrum of C4 at integration time of $4 \mathrm{~s}$.

As clearly seen in figures (4), (5) and (6), it is demonstrated that as the integration time is increased the $\mathrm{S} / \mathrm{N}$ ratio of Raman spectra get 
enhanced whereas the Raman bands are readily identifiable. For this measurement, the recorded signals enhanced with increasing the CCD integration time as the CCD camera accumulates the additions additively, rather than averaging the signal.

\section{Laser Power Influence}

As it was mentioned earlier, as the laser power is increased the Raman signal to noise ratio is considerably improved, the illustration to this phenomenon is that as the laser power is increased, it means the number of photons of the laser beam incident on the target sample is increased as a results, the number of inelastic Raman scattered photons is increased as such which eventually will enhance $\mathrm{S} / \mathrm{N}$ ratio of Raman spectra of sample to be detected. In order to demonstrate this, and to improve Raman $\mathrm{S} / \mathrm{N}$ ratio and trigger the weak Raman bands, the standoff Raman spectra of $\mathrm{C} 4$ were obtained for various laser powers i.e. $250 \mathrm{~mW}, 500 \mathrm{~mW}$ and $750 \mathrm{~mW}$ and the remaining parameter conditions kept fixed which are integration time of $400 \mathrm{~ms}$, standoff distance of $4 \mathrm{~m}$ and mass of $800 \mu \mathrm{g}$, and the measurement carried under dark condition. Firstly, Standoff measurements were taken for laser power of $250 \mathrm{~mW}$, as clearly shown in the Fig.(7), It is possible to record the most intense Raman bands at 885, 2948, 3000 and $3072 \mathrm{~cm}^{-1}$ while the remaining lower intensity bands in the spectroscopic region (12001600) $\mathrm{cm}^{-1}$ cannot be easily identified and are obscured by large background contributions recorded.

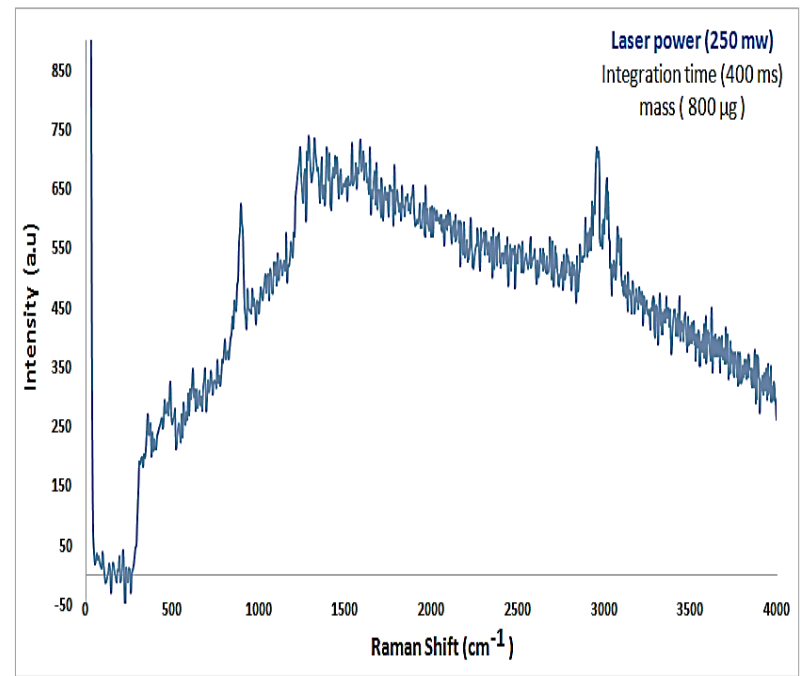

Fig.(7): Standoff Raman spectrum of C4 at laser power of $250 \mathrm{~mW}$.
Measuring the same sample with same conditions but with higher laser power of $500 \mathrm{~mW}$, the recorded spectrum at $500 \mathrm{~mW}$ shows that most intense bands are clearly enhanced, and the lower intensity bands within the region (1200-1600) $\mathrm{cm}^{-1}$ are enhanced and became distinguished but Raman bands at 1390, 1446 and $1590 \mathrm{~cm}^{-1}$ are still not so clearly defined as evidently shown in Fig.(8).

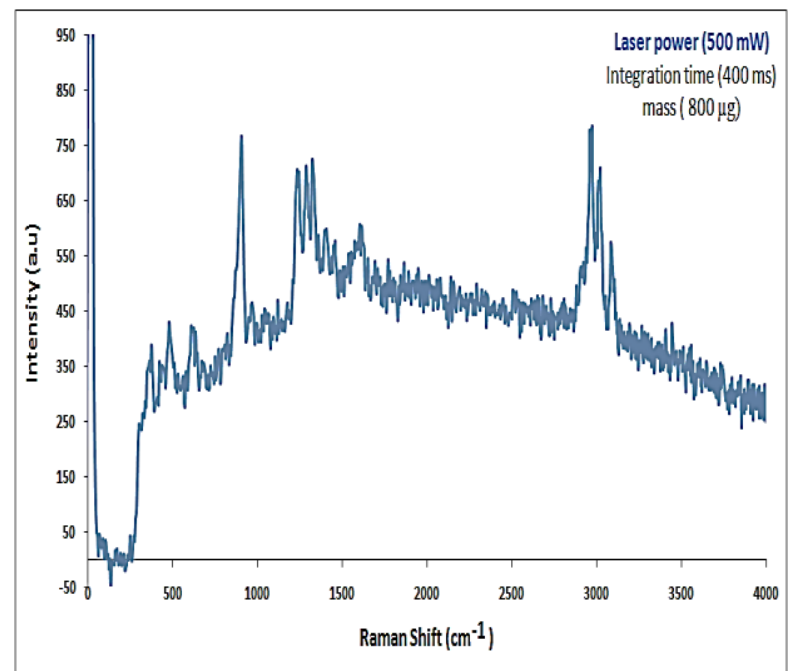

Fig.(8): Standoff Raman spectrum of C4 at laser power of $500 \mathrm{~mW}$.

The $\mathrm{S} / \mathrm{N}$ ratio of Raman spectrum may be improved and all characteristic of $\mathrm{C} 4$ substances are identified when the laser power set to be $750 \mathrm{~mW}$, as can be clearly seen in Fig.(9) such laser power was sufficient to clearly shows the ten characteristic Raman bands.

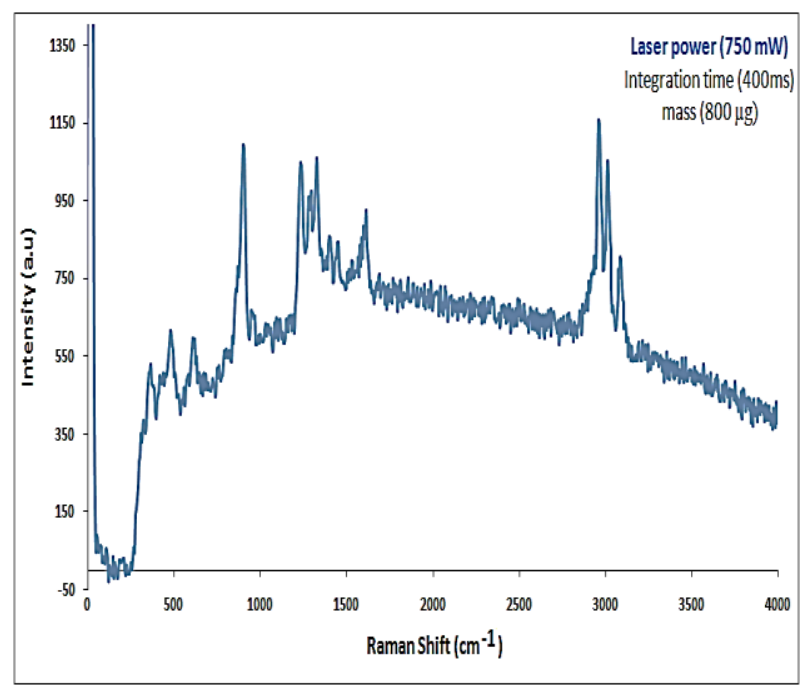

Fig.(9): Standoff Raman spectrum of C4 at laser power of $750 \mathrm{~mW}$. 
As clearly seen in Figs. (7), (8) and (9) it was demonstrated that there is a clear enhancement of Raman bands of $\mathrm{C} 4$ at relatively higher laser powers, in another word these results show that as the laser power is increased, a spectrum of $\mathrm{C} 4$ can be observed with relatively low background contribution to the spectrum.

\section{Standoff Raman Measurements under Partially Illuminated Environment PIE}

The standoff Raman detection of explosives takes place under different climatic conditions and in different level of ambient light, accordingly the detection may takes place in the daytime-outdoor environment and standoff detection in such environment is not possible to be achieved using continuous wave standoff Raman system and in some cases detection may carried out in the dark condition, in addition, the detection may takes place in the locations which are not directly exposed to sunlight where the existence of sunlight is not dominating, however such condition is considered as partially illuminated condition which represent low level of ambient light. In this part of study, the measurements carried out under partially illuminated environment where the two windows and the door of the laboratory kept open allowing the sunlight entering to the laboratory, and hence the laboratory became partially illuminated and such illumination is away below the level of illumination in the daytime-outdoor environment, however such measurements would allow to test the performance of standoff Raman spectroscopy system used in this study under partially illuminated environment. The measurements in such conditions are important because it is useful for several real-world applications including near nighttime, heavy cloudy days or locations such as airports, metro stations, train stations and in any buildings or places which prevent the direct exposure of sunlight, in another word, it is useful in any location where low ambient light is available.

In order to assess the applicability of SRS system for explosives detection in real world, the detection of $\mathrm{C} 4$ explosive under partially illuminated environment is achieved. The standoff Raman measurements have been performed on bulk amounts of $2 \mathrm{mg}$ at a standoff distance of $4 \mathrm{~m}$ away from the telescope using frequency doubled Nd:YAG laser at $532 \mathrm{~nm}$ excitation with laser power of $250 \mathrm{~mW}$ and various integration time for recording the spectrum. Standoff Raman spectrum of $\mathrm{C} 4$ detected at integration time of $1600 \mathrm{~ms}$ is shown in Fig.(10). As clearly seen in figure most of Raman bands are observed except two bands $1390 \mathrm{~cm}^{-1}$ and $1446 \mathrm{~cm}^{-1}$ which were not possible to be distinguished, therefore it can be said that the $\mathrm{S} / \mathrm{N}$ ratio of Raman spectrum is good enough but the Raman signal is much weaker than the Raman signal obtained under dark condition as shown in prior Fig.(1) and this is due to the interference of high amount of ambient background noise under partially illuminated environment.

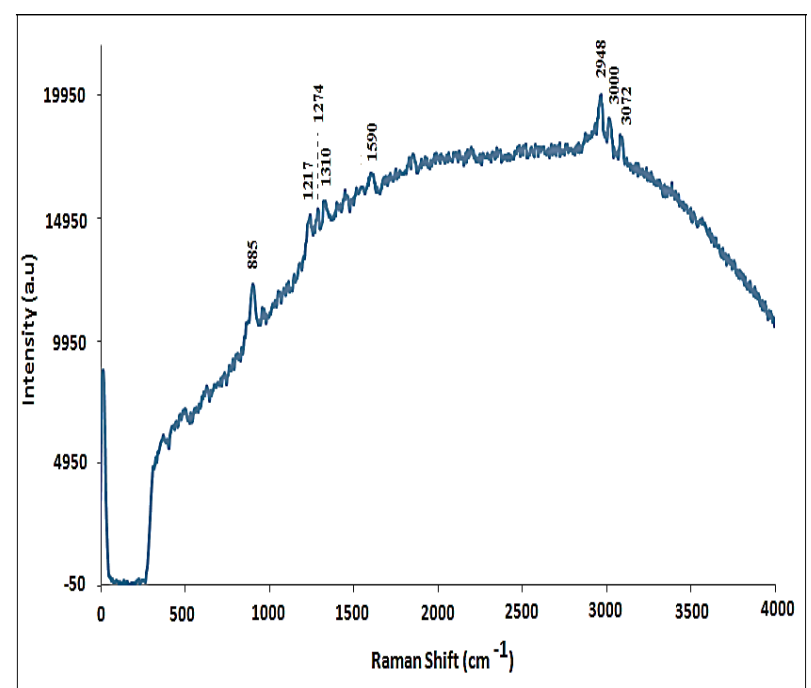

Fig.(10): Standoff Raman spectrum of C4 under PIE at IT of $1600 \mathrm{~ms}$.

As shown in the previous part of study for measurements under dark conditions, it was proved that as the integration time is increased the Raman $\mathrm{S} / \mathrm{N}$ ratio is significantly enhanced where $\mathrm{S} / \mathrm{N}$ ratio at the integration time of $4 \mathrm{~s}$ Fig.(6) was much enhanced compared with $1 \mathrm{~s}$ Fig.(5) and $400 \mathrm{~ms}$ Fig.(4). In this part of study, the influence of integration time increment has also been studied. Firstly it was expected that the integration time increment factor under partially illuminated environment will enhance the $\mathrm{S} / \mathrm{N}$ in the same efficiency occurring in the dark conditions, therefore based on that anticipation, the integration time increased from $1600 \mathrm{~ms}$ to $1800 \mathrm{~ms}$ to further 
enhance $\mathrm{S} / \mathrm{N}$ ratio of $\mathrm{C} 4$ spectrum, but as shown in Fig.(11) once the C4 Raman spectrum is recorded, it is noticed that the $\mathrm{S} / \mathrm{N}$ ratio of Raman spectrum is not enhanced on the contrary, it is degraded and hence the Raman bands are no longer identified except one band at $885 \mathrm{~cm}^{-1}$ which is not enough to identify $\mathrm{C} 4$ substance, therefore it is demonstrated that enhancing the $\mathrm{S} / \mathrm{N}$ of Raman spectrum of $\mathrm{C} 4$ via integration times is limited only to integration time of $1600 \mathrm{~ms}$ and below and any other increment in integration time above $1600 \mathrm{~ms}$ is no longer useful under partially illuminated environment and the reasoning to this phenomenon is as follow, as the integration time is increased the ambient sunlight photons entering through the telescope and finally to the detector will be much more than inelastic Raman scattered photons from the sample as a results the ambient background is dominating and the Raman bands is no longer observed.

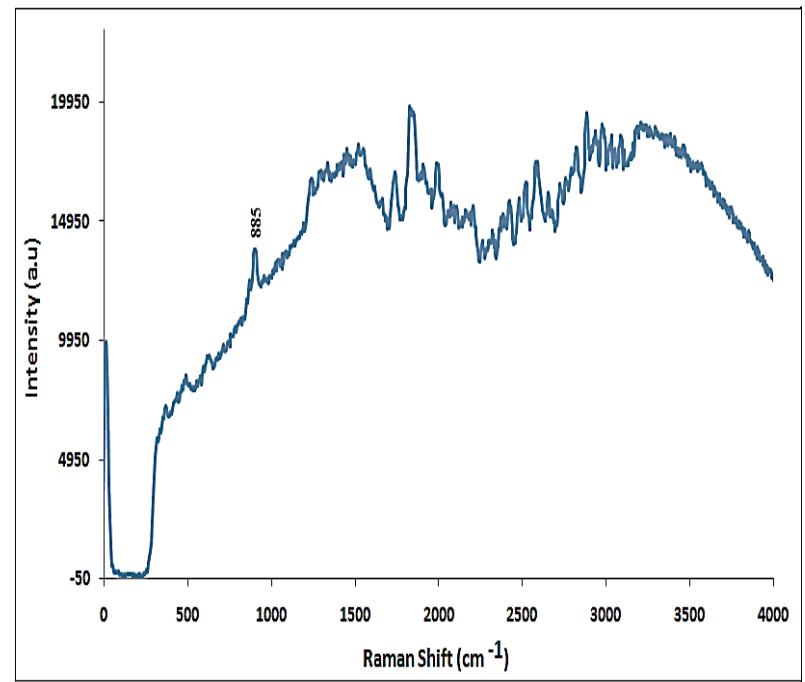

Fig.(11): Standoff Raman spectrum of C4 under PIE at IT of $1800 \mathrm{~ms}$.

For measurements under well illuminated condition it necessitate to use pulsed standoff Raman spectroscopy system instead of continuous wave standoff Raman system to suppress the huge amount of background noise.

\section{Conclusions}

The conclusion of the present paper that the designed standoff Raman spectroscopy system is proved to be capable of detecting $\mathrm{C} 4$ explosive substance under dark conditions at distance of 4 meters. The standoff Raman spectra of standard samples Carbon tetrachloride $\mathrm{CCL}_{4}$ and Acetone verified the performance of Raman system.

It has been demonstrated that integration time and laser power increment substantially enhance Raman $\mathrm{S} / \mathrm{N}$ ratio for $\mathrm{C} 4$ substance and enable detection of low intensity bands in Raman spectrum.

The capability of continuous wave SRS system is demonstrated in detecting explosives under partially illuminated environment and the measurements performed on $\mathrm{C} 4$ substance at distance of $4 \mathrm{~m}$. In addition, it was discovered that under such illumination, increasing the integration time will not continuously be enhancing Raman signal for long range of time but instead enhancement is limited to short range of time which was 1600 ms for this study where the Raman bands above $1600 \mathrm{~ms}$ are overmusked by background noise.

\section{References}

[1] Gardiner D. J., "Practical Raman Spectroscopy. New York: Springer-Verlag, 1989.

[2] Schubert, H., Kuznetsov, A. Eds.; (a) "Vapour and Trace Detection of Explosives for Anti-Terrorism Purpose", Kluwer Academic Publishers: Dordrecht, the Netherland, 2003. (b) "Detection and Disposal of Improvised Explosives", Springer: Dordrecht, the Netherland, 2005.

[3] Ortiz-Rivera W., Pacheco-Londono L. C., Hernandez-Rivera S. P., "Remote Continuous Wave and Pulsed Laser Raman Detection of Chemical Warfare Agents Simulants and Toxic Industrial Compounds", Sens Imageing 11, 131-145, 2010.

[4] Garcia C. S., Abedin M. N., Sharm S.K., Misra A. K., Ismail S., Singh U., Refaat T. F., Ali H. E., Sandford S., "Remote pulsed laser Raman spectroscopy system for detecting water, ice, and hydrous minerals", Hawaii Inst. of Geophysics, Honolulu, HI96822. 2005

[5] Wakabayashi K., Nakayama T., Koshi Y. M., "Temporal change of Raman spectra of carbon tetrachloride under laser-driven 
shock compression", AIP Conference Proceedings, 955(1), 1267-1270. 2007.

[6] Hernández Rivera S. P., Ortiz Rivera W., "Standoff Raman system for detection of explosives", Puerto Rico: University of Puerto Rico 2008.

[7] Torres P., Mercado L., Cotte I., Hernandez S. P., Mina N. Santana A., Chamberlain R. T., Lareau R., Castro M. E. "Vibrational Spectroscopy Study of $\beta$ and $\alpha$ RDX Deposits", J. Phys. Chem. B, 108, 8799 8805, 2004.

[8] Lin-Vien D., Colthup N. B., Fateley W. G., Grasselli J. G., "The Handbook of Infrared and Raman Characteristic Frequencies of Organic Molecules", Academic Press: San Diego, CA, 1991.

[9] Pacheco-Londono L.C., Ortiz-Rivera W., Primera-Pedrozo M. O., Hernández-Rivera S. P., "Vibrational spectroscopy standoff detection of explosives". Anal Bioanal Chem. 395, 323-335, 2009. 